\title{
Evaluation of the role of $R E T$ polymorphisms/haplotypes as modifier loci for MEN 2, and analysis of the correlation with the type of RET mutation in a series of Spanish patients
}

\author{
RAQUEL MARÍA FERNÁNDEZ ${ }^{1}$, ELENA NAVARRO², GUILLERMO ANTIÑOLO ${ }^{1}$, \\ MACARENA RUIZ-FERRER $^{1}$ and SALUD BORREGO ${ }^{1}$ \\ ${ }^{1}$ Unidad Clínica de Genética y Reproducción, ${ }^{2}$ Unidad Clínica de Endocrinología, \\ Hospitales Universitarios Virgen del Rocío, Sevilla, Spain
}

Received September 30, 2005; Accepted November 22, 2005

\begin{abstract}
Multiple endocrine neoplasia type 2 (MEN 2) is an autosomal dominant cancer syndrome, which is divided into three subtypes: MEN 2A, MEN 2B and familial medullary thyroid cancer (FMTC). Approximately $92 \%$ of MEN 2 cases are caused by mutations in exons $10,11,13-16$ of the RET proto-oncogene. There exists inter- and intra-familial phenotypic variability among the MEN 2 families, even when the disease is caused by the same RET mutation, suggesting a role for genetic modifiers, such as polymorphisms/haplotypes. We have sought to determine the frequency and position of RET germline mutations in a cohort of 114 Spanish probands with any sign of MEN 2, and to search for putative modifier loci. Mutational screening of RET revealed 9 different mutations, present in 26 of the 114 probands (22.8\%). In addition, distributions of $8 R E T$ polymorphisms and the haplotypes comprising them, were studied in the context of the families positive for RET mutational screening, in order to evaluate them as genetic modifiers. The relationship between RET mutation type and presence of a polymorphism/haplotype was analyzed. The relationship between the presence of pheochromocytoma (PC) and/or hiperparathyroidism (HPT) in carriers of the same RET mutation, and the genotype for the specific variants was also studied. The results derived from those analyses revealed no associations of any variant/haplotype to a specific mutation or to the clinical presentation. Nevertheless, these observations do not permit us to exclude the possible role of other variants in RET or other related genes, in the final presentation of the disease.
\end{abstract}

Correspondence to: Dr Salud Borrego, Unidad Clínica de Genética y Reproducción, Hospitales Universitarios Virgen del Rocío, Avda. Manuel Siurot s/n, 41013 Sevilla, Spain

E-mail: salud.borrego.sspa@juntadeandalucia.es

Key words: MEN 2, medullary thyroid cancer, RET proto-oncogene, genetic modifier, polymorphism, haplotype

\section{Introduction}

Medullary thyroid carcinoma (MTC) is a tumour of the thyroid $\mathrm{C}$ cells which occurs as a part of the heritable multiple endocrine neoplasia type 2 (MEN 2, OMIM 171400) in 25\% of cases, and sporadically in the remaining cases. MEN 2 is a cancer syndrome transmitted as an autosomal dominant trait, and presenting with a high degree of penetrance and variable clinical expression. MEN 2 is divided into three sub-types depending on the organs involved: MEN 2A, MEN 2B and familial MTC (FMTC). MEN 2A comprises the classic triad of MTC, pheochromocytoma (PC) in approximately $50 \%$ of cases, and hyperparathyroidism (HPT) in 15-30\% of cases $(1,2)$. The features of MEN 2B are similar to those of MEN $2 \mathrm{~A}$, except that the average age of tumour onset is 10 years earlier, clinically evident parathyroid disease is absent, and developmental abnormalities such as ganglioneuromatosis, medullated corneal nerves, and marfanoid habitus are present $(2,3)$. Finally, FMTC comprises families with MTC as their only disease phenotype $(2,4)$.

The three forms of MEN 2 are caused by specific germline mutations of the RET proto-oncogene, which encodes a receptor tyrosine kinase expressed in derivatives and tumours of neural crest origin (2). In $>98 \%$ of MEN 2A families, the affected members have a RET missense mutation in one of the five cysteine codons of exon $10(609,611,618$, and 620) and exon $11(634)(5,6)$. In each case, these changes result in replacement of a critical cysteine residue, normally involved in the intramolecular disulphide bonds that determine the tertiary structure of RET, so that the outcome is dimerisation and constitutive activation of the receptor (7-9). It has been reported that approximately $87 \%$ of MEN 2A mutations affect codon 634 and the most frequent substitution at this codon is a cysteine to arginine change (C634R), found in $>50 \%$ of cases (5). In MEN 2A Spanish families high prevalence (73\% of cases) was denoted of the C634Y mutation (10), which would suggest a founder effect of this mutation in the Spanish population. Many of the mutations responsible for MEN 2A have been found in $>80 \%$ of FMTC families as well. FMTC has also been shown to be associated with mutations in the tyrosine kinase domain of RET, mainly 
Table I. PCR conditions for RET mutational screening.

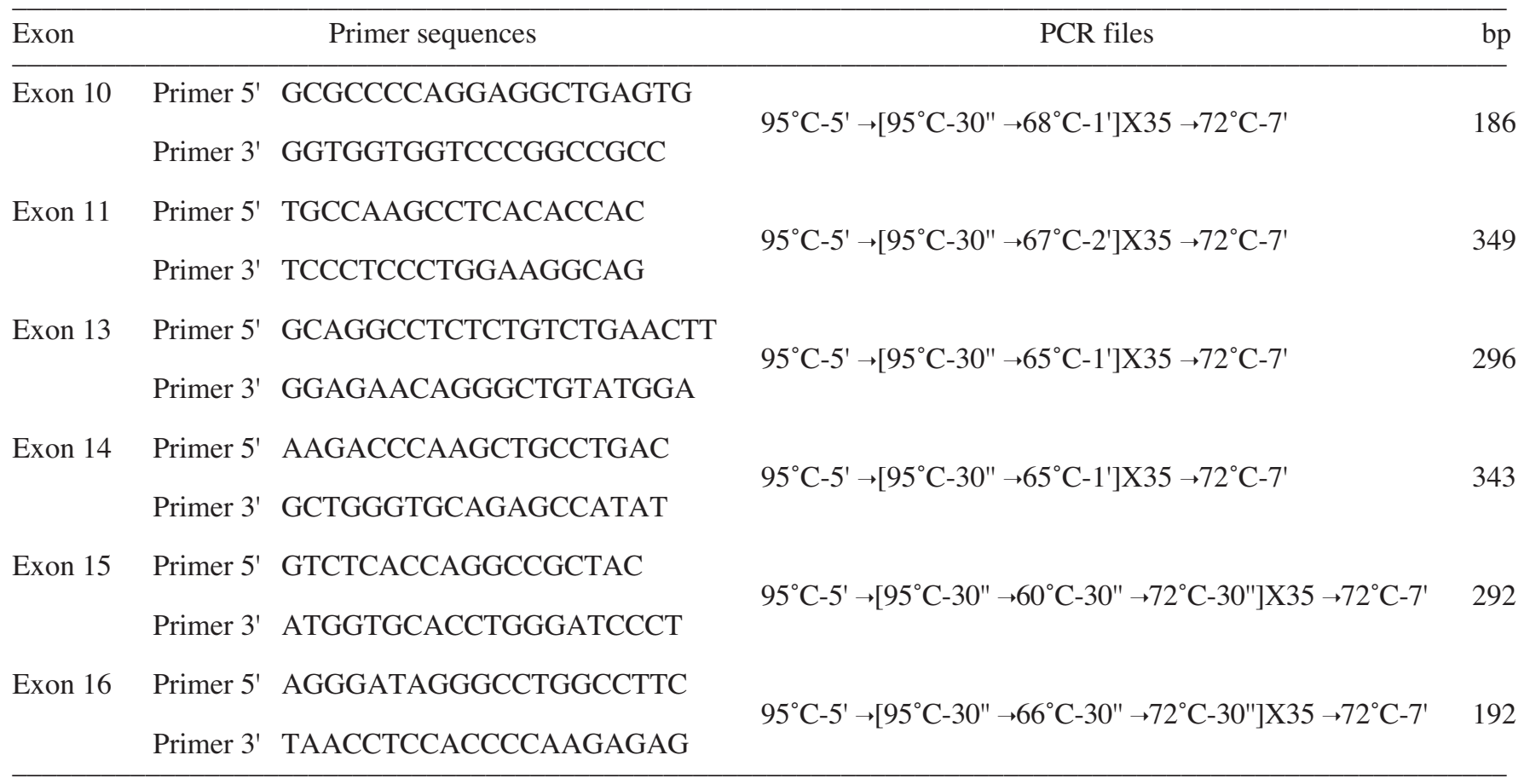

affecting codons 768 (exon 13), 804 (exon 14) or 891 (exon 15) $(2,6)$. The mechanisms by which each of these individual mutations activates RET have not been functionally demonstrated; however, they are predicted to alter either ATP or RET substrate binding (11-13). Regarding MEN 2B, it is associated primarily with a single missense mutation of codon 918 in exon 16 (M918T) which is found in $>90 \%$ of all reported cases, while the mutations affecting codons 883 (exon 15) or 922 (exon 16) occur in a small number of cases $(14,15)$. These mutations result in RET proteins with altered substrate specificity $(6,16)$.

It has been widely demonstrated that there exist inter- and intra-familial phenotypic variability among MEN 2A families regarding the presence of PC and/or HPT, and among MEN 2A/FMTC families with respect to the age of onset, even when the disease is caused by the same germline RET mutation. Such variations suggest a role for genetic modifiers, which may also work through quantitative effect (17), such as the presence of additional somatic mutations or specific polymorphisms/haplotypes. The possible role of two RET polymorphisms, G691S (c.2071C $\rightarrow \mathrm{A}$, exon 11) and S904S (c.2712C $\rightarrow \mathrm{G}$, exon 15) has been reported on the age of onset of MEN 2A $(18,19)$. Robledo et al analyzed a total of 35 unrelated MEN 2A families and found that the homozygous patients for these polymorphisms were, on average, 10 years younger at diagnosis compared with heterozygous and wild-type homozygous (19). Some other reports on FMTC families have speculated about the possible involvement of certain RET polymorphisms, such as L769L (c.2307T $\rightarrow \mathrm{G}$, exon 13) or S836S (c. 2439C $\rightarrow \mathrm{T}$, exon 14), in the reduced penetrance of the disease among carriers of mutations affecting the 804 codon $(20,21)$. Results derived from a recent study revealed the over-representation or exclusive presentation of some variants within the GFRA4 gene (encoding a co-receptor of RET) in MEN 2 patients, when comparing with their healthy relatives, thus suggesting a putative modifying role for such polymorphisms in MEN 2 (22).

The aim of the present study was to determine the frequency and position of RET germline mutations in a large cohort of Spanish probands with any sign of MEN 2 syndromes, as well as to evaluate several RET polymorphisms/haplotypes as modifier loci. With this objective, we selected $8 R E T$ variants distributed along the whole genomic sequence of the gene, located in the promoter region $(-200 \mathrm{~A} \rightarrow \mathrm{G},-196 \mathrm{C} \rightarrow \mathrm{A})$, intron 1 (IVS1-1463T $\rightarrow$ C, IVS1-1370C $\rightarrow$ T, IVS1-126G $\rightarrow$ T), and exons 11, 14 and 15 (G691S, S836S and S904S).

\section{Patients and methods}

Patients. In this study we analyzed a total of 114 unrelated probands from Spain ascertained and serviced by our hospital, presenting with any sign of MEN 2 syndromes. Among the 114 patients, 100 presented with MTC and they were clinically and biochemically characterized and classified as sporadic MTC (sMTC, 73 patients), FMTC (8 patients), MEN 2A (16 patients) and MEN 2B (3 patients). In one of the MEN 2A families, such phenotype co-segregates with Hirschsprung disease (HSCR, OMIM 142623), and this pedigree is described in detail in previous reports $(23,24)$. In the remaining 14 individuals, MTC was absent but they presented with PC (10 patients), HPT (2 patients) or PC + HPT (2 patients). They were also analyzed and included in this study since PTC and/or HPT can constitute the first manifestation of the MEN 2A syndrome in some cases (25). In all the cases, diagnosis of MTC, HPT and PC was based on documented pathological examination. An informed consent was obtained from all the participants for clinical and molecular genetic 
Table II. Detected mutations in the $26 R E T^{+}$families.

\begin{tabular}{|c|c|c|c|c|c|c|}
\hline Fam. code & Exon & Codon & Base change & Amino acid change & Phenotype & $R E T^{+} /$family members studied \\
\hline 1 & 11 & 634 & TGC/TAC & Cys/Tyr & MEN 2A & $22 / 44$ \\
\hline 2 & 11 & 634 & TGC/TAC & Cys/Tyr & MEN 2A & $2 / 11$ \\
\hline 4 & 11 & 634 & TGC/CGC & Cys/Arg & FMTC & $2 / 4$ \\
\hline 9 & 11 & 634 & TGC/TAC & Cys/Tyr & MEN 2A & $8 / 28$ \\
\hline 11 & 11 & 634 & TGC/TAC & Cys/Tyr & MEN 2A & $2 / 4$ \\
\hline 16 & 15 & 891 & TCG/GCG & Ser/Ala & FMTC & $3 / 8$ \\
\hline 19 & 11 & 634 & TGC/TAC & Cys/Tyr & MEN 2A & $2 / 12$ \\
\hline 20 & 11 & 634 & TGC/CGC & Cys/Arg & MEN 2A & $2 / 3$ \\
\hline 23 & 11 & 634 & TGC/TAC & Cys/Tyr & MEN 2A & $5 / 15$ \\
\hline 24 & 10 & 618 & TGC/CGC & Cys/Arg & FMTC & $3 / 4$ \\
\hline 27 & 11 & 634 & TGC/TAC & Cys/Tyr & MEN 2A & $3 / 8$ \\
\hline 31 & 11 & 634 & TGC/CGC & Cys/Arg & FMTC & $6 / 11$ \\
\hline 32 & 10 & 620 & TGC/TCC & Cys/Ser & MEN 2A + HSCR & $2 / 10$ \\
\hline 35 & 11 & 634 & TGC/TAC & Cys/Tyr & MEN 2A & $8 / 24$ \\
\hline 40 & 11 & 634 & TGC/TAC & Cys/Tyr & MEN 2A & $2 / 4$ \\
\hline 44 & 11 & 634 & TGC/TAC & Cys/Tyr & MEN 2A & $6 / 11$ \\
\hline 46 & 10 & 600 & CGC/CAG & Tyr/Gln & sMTC & $4 / 7$ \\
\hline 47 & 16 & 918 & ATG/ACG & Met/Thr & MEN 2B & $0 / 7$ \\
\hline 48 & 11 & 634 & TGC/CGC & Cys/Arg & MEN 2A & $1 / 5$ \\
\hline 51 & 11 & 634 & TGC/TAC & Cys/Tyr & MEN 2A & $1 / 1$ \\
\hline 73 & 16 & 918 & ATG/ACG & Met/Thr & MEN 2B & $0 / 2$ \\
\hline 74 & 13 & 768 & GAG/GAT & Glu/Asp & sMTC & $0 / 4$ \\
\hline 91 & 11 & 634 & TGC/TAC & Cys/Tyr & MEN 2A & $1 / 6$ \\
\hline 109 & 10 & 609 & TGC/TCT & Cys/Ser & MEN 2A & $1 / 1$ \\
\hline 121 & 11 & 634 & TGC/CGC & Cys/Arg & $\mathrm{PC}$ & $0 / 2$ \\
\hline 137 & 16 & 918 & ATG/ACG & Met/Thr & MEN 2B & $0 / 2$ \\
\hline
\end{tabular}

The family phenotype refers to the initial diagnosis based on clinical/biochemical results and study of familial history, previous to the molecular analysis.

studies conforming to the tenets of the Declaration of Helsinki.

RET mutational screening. Genomic DNA was extracted according to standard protocols (26) from all the individuals tested. As recommended by the standard guidelines for diagnosis of MEN 2 (27), molecular analysis of exons 10, 11, $13,14,15$ and 16 of $R E T$ were performed in all the 114 index patients by direct sequencing in either an ALF express semiautomated machine or a MegaBACE automated sequencer (Amersham Biosciences). Primers and PCR conditions are shown in Table I. In addition, in mutation positive cases, their relatives at risk of the disease were also screened.

Evaluation of RET variants as modifier loci for MEN $2 \mathrm{~A}$ or $F M T C$. A total of 8 RET sequence variants were genotyped in the 259 available members of our 16 MEN 2A and 8 FMTC families. In addition, we also included the 9 available members of the families of the 2 initially considered sMTC and 1 PTC patients, who finally resulted positive for $R E T$ mutational screening.
The 2 polymorphisms located within the promoter region of the gene $(-200 \mathrm{~A} \rightarrow \mathrm{G}$ and $-196 \mathrm{C} \rightarrow \mathrm{A})$, and the intronic variants IVS1-1463T $\rightarrow$ C (rs2505533) and IVS1-1370C $\rightarrow$ T (rs2505532) were genotyped using the 'fluorescence resonance energy transfer' (FRET) technology, as previously described $(28,29)$. The SNPs located in intron 1 (IVS1-126G $\rightarrow \mathrm{T}, \mathrm{rs} 2565206)$, exon 11 (G691S, rs1799939), exon 14 (S836S, rs1800862) and exon 15 (S904S, rs1800863) were genotyped by differential restriction digestion with NlaIII, BanI, AluI and $R s a$ I enzymes respectively, using the protocols previously described (28,30-34).

Allelic and genotypic distribution of the 8 RET polymorphisms and the haplotypes comprising them, were analyzed in the context of the families. The generation of haplotypes was possible due to the availability of DNA from some firstdegree relatives of the patients, allowing us to reconstruct and compare the shared versus non-shared alleles. In each family, the frequencies of each variant/haplotype were calculated and compared among the affected patients and their healthy relatives. 


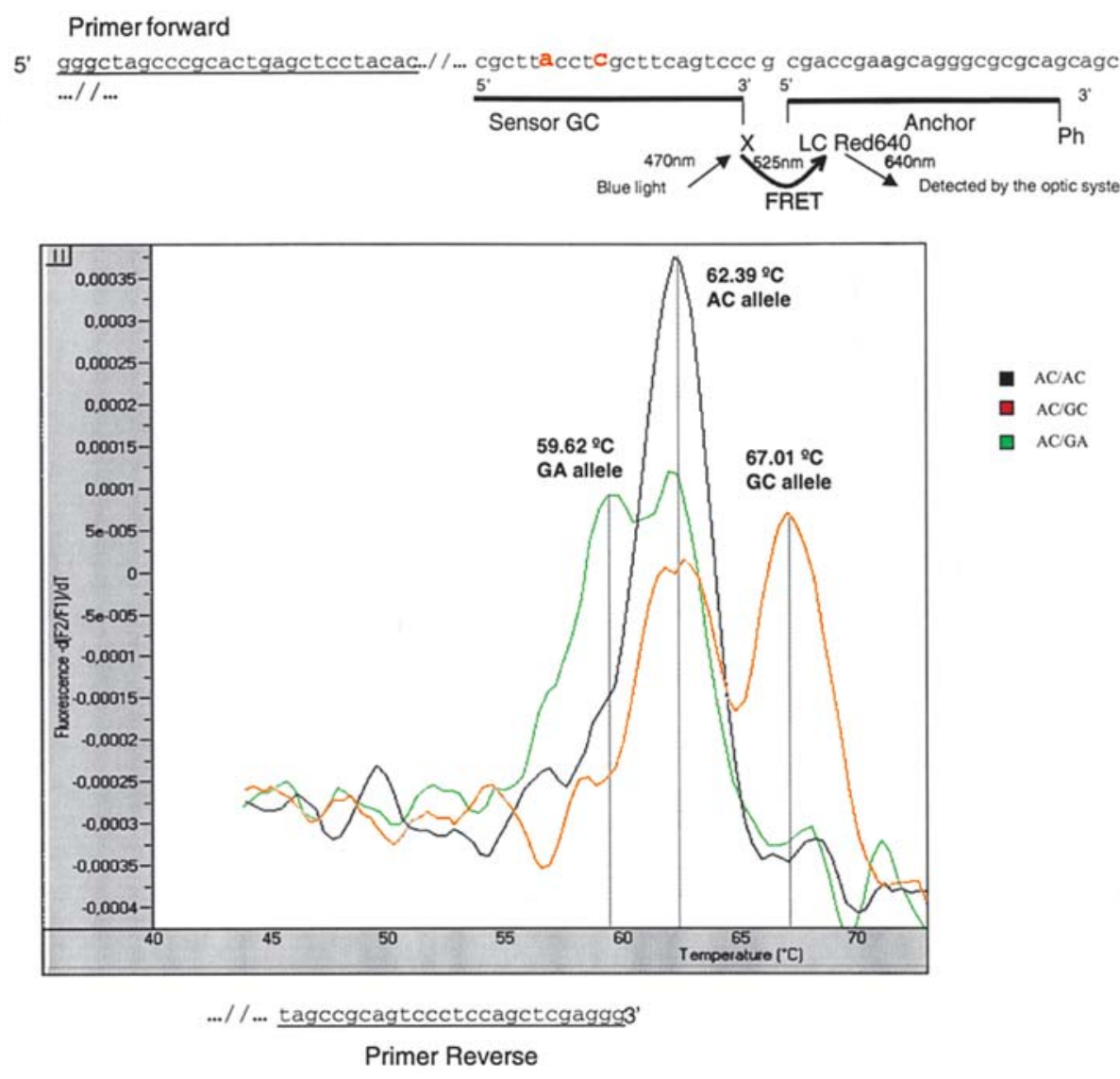

Figure 1. FRET pattern for the simultaneous detection of the $R E T$ variants $-200 \mathrm{~A} \rightarrow \mathrm{G}$ and $-196 \mathrm{C} \rightarrow \mathrm{A}$.

We have analyzed the relationship between RET mutation type and presence of a given RET polymorphism or haplotype. The relationship between the presence of PC and/or HPT in carriers of the same RET mutation, and the genotype for the specific variants was studied. Comparisons were performed using either Chi-square analysis with the Yate's correction, or Fisher's two-tailed exact test when appropriate. Statistical significance was set at $\mathrm{p}<0.05$.

\section{Results}

We screened a total of 114 unrelated probands, comprising 16 MEN 2A, 3 MEN 2B, 8 FMTC, 73 sMTC, 10 PC, 2 HPT and $2 \mathrm{PC}+\mathrm{HPT}$ patients, and found RET germline mutations in 26 of them (Table II). Among the familial MTC cases, only 4 clinically diagnosed FMTC families, each with 2 members affected from MTC, remain with no identified RET mutation. We detected 9 different types of mutations in exons 10 (R600Q, C609S, C618R, C620S), 11 (C634R, C634Y), 13 (E768D), 15 (S891A) and 16 (M918T) of RET, while in exon 14 no mutations were found (Table I). The most prevalent mutations were C634Y (detected in 12 of the MEN 2A families), and C634R (present in 2 MEN 2A and 2 FMTC pedigrees, as well as in a previously considered sporadic PC patient). The C620S was the cause of the MEN2A phenotype in the MEN 2A/HSCR family, and the C609S mutation was detected in another MEN 2A family. We also found two FMTC families with the C618R and the S891A mutations respectively, and another patient initially diagnosed as sporadic
MTC carried E768D. In addition, when performing mutational screening of exon 10 in one apparently sMTC patient, we detected the R600Q variant, not classically considered as a typical RET oncogenic mutation. Finally, the M918T was found as a de novo mutation in the $3 \mathrm{MEN} 2 \mathrm{~B}$ pedigrees.

Subsequently, we proceeded to perform molecular analysis in the relatives of the 26 RET mutation carriers. Among 238 relatives at risk of carrying the mutation, 86 resulted positive for this test, 43 with clinical manifestations of MEN 2 and 43 still without any sign (Table II).

The 8 RET variants were genotyped in the 268 available members of the 16 MEN 2A, 4 FMTC, 2 apparently sMTC, and 1 apparently sporadic PTC families which resulted positive for RET mutational screening. We used previously published genotyping methods, but made a modification on the test for the 2 variants of the promoter, since we employed a sensor probe different to that previously reported in Fernandez et al (29) (Fig. 1). We verified that the presence of any of the polymorphisms, in both homozygosity or heterozygosity, was absolutely independent of the specific pathogenic mutation of $R E T$ in such families. Subsequently we proceeded to construct haplotypes constituted by the combination of the alleles present at each polymorphic RET loci, and found 9 different specific combinations (Table III), although some of them were very infrequent. Strong co-segregation between some of the variants tested was found (Table III). Our intention was to investigate the specific RET allele associated with the oncogenic mutation in each family, in order to elucidate if the pedigrees sharing the same mutation might descend from 
Table III. Definition of the haplotypes comprising 8 specific $R E T$ variants found in our families.

\begin{tabular}{lcccccccccc}
\hline$R E T$ variants & 0A & 0B & 0C & $1 \mathrm{~A}$ & $1 \mathrm{~B}$ & $1 \mathrm{C}$ & $2 \mathrm{~A}$ & $2 \mathrm{~B}$ & $2 \mathrm{C}$ & 4 \\
\hline$-200 \mathrm{~A} \rightarrow \mathrm{G}$ & - & - & - & + & + & + & + & + & + & + \\
$-196 \mathrm{C} \rightarrow \mathrm{A}$ & - & - & - & + & + & + & - & - & - & + \\
$\mathrm{IVS1-1463T \rightarrow C}$ & - & - & - & + & + & + & + & + & + & + \\
$\mathrm{IVS1} 1370 \mathrm{C} \rightarrow \mathrm{T}$ & - & - & - & + & + & + & - & - & - & + \\
IVS1-126G $\rightarrow \mathrm{T}$ & - & - & - & - & - & - & + & + & + & + \\
$\mathrm{G} 691 \mathrm{~S}$ & - & + & - & - & + & - & - & + & - & - \\
$\mathrm{S} 836 \mathrm{~S}$ & - & - & + & - & - & + & - & - & + & - \\
S904S & - & + & - & - & + & - & - & + & - & - \\
\hline
\end{tabular}

Some of the haplotypic combinations were very infrequent, such as $0 \mathrm{~B}, 0 \mathrm{C}$ or 4 . Haplotype analysis in the context of our families reveled strong cosegregation among $-200 \mathrm{~A} \rightarrow \mathrm{G} / \mathrm{IVS} 1-1463 \mathrm{~T} \rightarrow \mathrm{C},-196 \mathrm{C} \rightarrow \mathrm{A} /$ IVS1-1370C $\rightarrow$ T and G691S/S904S.

Table IV. RET haplotypes associated to each kind of oncogenic mutation.

\begin{tabular}{lccc}
\hline Mutation & Haplotype & $\begin{array}{c}\text { No. of } \\
\text { families }\end{array}$ & $\begin{array}{c}\text { Family } \\
\text { phenotype }^{\mathrm{a}}\end{array}$ \\
\hline Cys634Tyr & 0A & 7 & MEN 2A \\
& 2A & 3 & MEN 2A \\
& 1B & 1 & MEN 2A \\
Cys634Arg & 1B & 1 & MEN 2A \\
& 0A & 2 & 1 MEN 2A and 1 FMTC \\
& 2A & 1 & 1 MEN 2A and 1 PTC \\
& & & FMTC
\end{tabular}

aThe family phenotype refers to the initial diagnosis based on clinical/biochemical results and study of familial history, previous to the molecular analysis. ?, in this family the RET haplotype could not be deduced because the data were not informative.

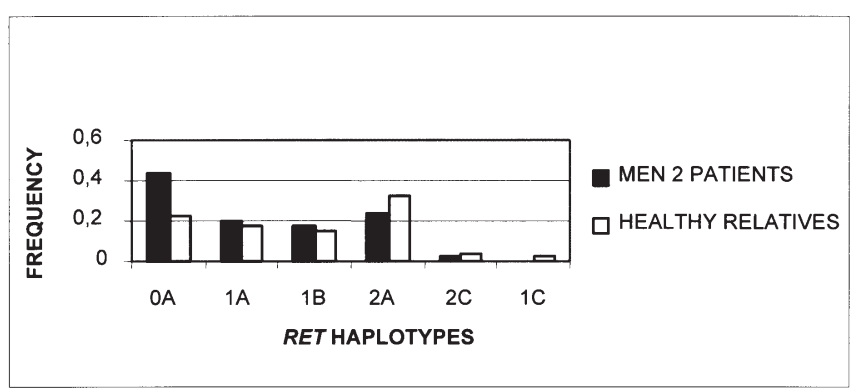

Figure 2. Distribution of RET haplotypes in MEN 2 patients and their healthy relatives.

common ancestors. However, as shown in Table IV, we observed that the most frequent mutations present in our families, C634Y and C634R, were associated with several different haplotypes. We also found that the allelic and
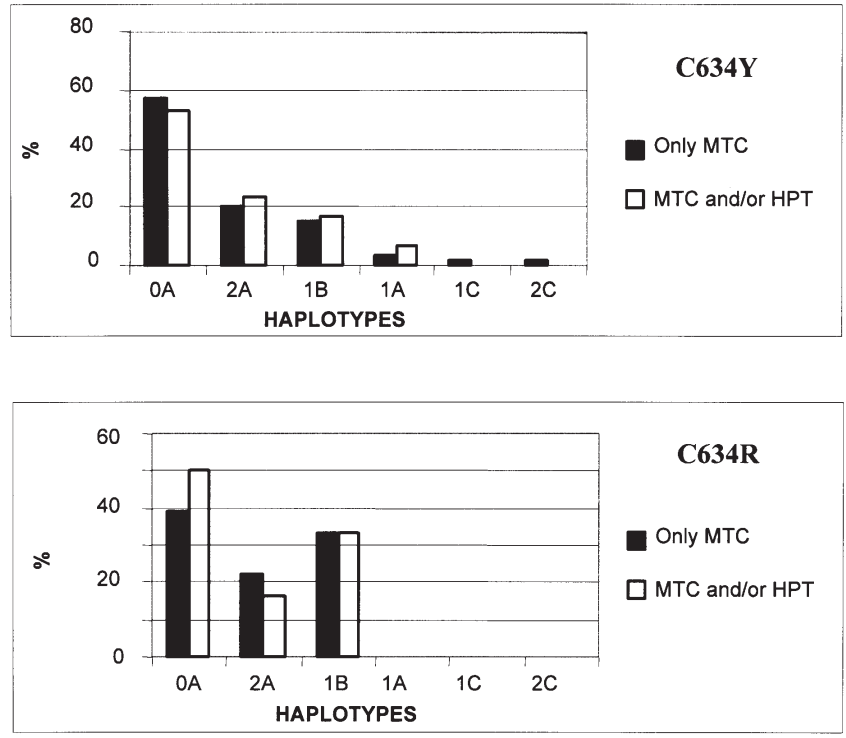

Figure 3. Haplotypic distribution in patients carrying the same RET mutation with a different clinical presentation.

haplotypic distribution was similar among our MEN 2 patients and their healthy relatives in the context of each family, and also in a global sense (Fig. 2). The values of frequency were very similar to those obtained in the general Spanish population $(28,29,31,32)$.

In addition, we proceeded to analyze the distribution of the RET variants and haplotypes for those 4 FMTC families with no oncogenic mutation identified, in order to evaluate their association with the manifestation of the disease. This analysis led us to observe that the 4 FMTC families were linked to RET, since in each pedigree the affected members shared the same specific haplotype. However, when comparing among the 4 families, we found variability regarding the specific haplotype which seemed to segregate with the disease (2 families with haplotype 1A, 2 with haplotype 1B, and 2 with haplotype 0A).

By performing the genotype-phenotype analysis we confirmed the association of the mutations affecting codon 634 and the presence of PTC or PTC + HPT in the families (Fisher's exact test with two tails, $\mathrm{p}=0.03$ ). Nevertheless, there existed inter- and intra-familial phenotypic variability among the patients carrying one of those specific RET mutations (Table IV; C634Y or C634R). Thus we proceeded to analyze and compare the distribution of the polymorphisms/haplotypes in those groups of patients, in order to evaluate them as modifier elements. However, a random transmission, either cis or trans, of the 8 variants tested was found, so that no association was observed between any polymorphism and the presence of PC and/or HPT. In addition no specific haplotype was found to correlate with the presentation of the disease (Fig. 3).

\section{Discussion}

In this report we present a comprehensive study of molecular genetic screening of Spanish families with any sign typical of MEN 2 syndromes. Some parts of the families included 
here have been partially presented in previous reports $(10,23,24,35,36)$. Germline mutations were found in $22.8 \%$ of the probands of our MTC families, and in 1 patient presenting with PC. Our results are in concordance with the data stating that up to $25 \%$ of MTC cases are inherited $(2,5,6)$. In FMTC families our detection rate was only $50 \%$ (4 out of 8 families), probably due to the application of milder clinical criteria for this group. For our 4 FMTC unsolved families, the disease could still be a part of an inherited syndrome where the mutation may reside in other exons of $R E T$, just as it occurs with some FMTC pedigrees previously published $(37,38)$. In this sense, our haplotype analysis confirms that all these families are indeed linked to RET. However, the inheritance of some mutations lying in other genes cannot be completely excluded.

Again our data seem to confirm the association of mutations affecting codon 634 and the presence of phaeochromocytoma or parathyroid disease observed in the International RET Mutation Consortium series (5). As previously postulated (39), this apparent association might be due not to a direct effect of the resultant amino acid substitution on susceptibility to PC or HPT disease, but to the effect of a founder chromosome which carries both the codon 634 mutation, and another tightly linked variant determining the probability of PC/HPT involvement. However, our results derived from the haplotype analysis revealed that each of the 634 mutations is represented by more than one haplotype, thus suggesting that the oncogenic mutations themselves are responsible for the final phenotype in the families.

On the other hand, with this haplotype analysis we also sought to investigate the phenotypic inter- and intra-familial variability among patients carrying the same mutation at codon 634. Because PTH and/or PC were not a consistent feature in all the patients who carried mutations affecting codon 634 , there must exist other factors, which might modify the expression of the mutation with respect to $\mathrm{PC} / \mathrm{PTH}$ manifestations. It is supposed that if the presence of a polymorphism correlates (or associates) with a change in phenotype, it would be plausible to infer that such polymorphism indeed acts as a modifier. Thus, we proceeded to analyze a selection of RET variants as putative modifier loci. Among those polymorphisms we had selected G691S and S904S because of their previous definition as genetic modifiers of MEN 2A by Robledo et al (19). They found a strong correlation between both variants and the age of onset of MEN 2A. It was then proposed that such specific effect of G691S probably could be attributable to some cooperative action on RET-dimerization with the oncogenic mutations. Alternatively, it was also postulated that G691S might create a new site susceptible to serine-phosphorylation affecting to some extent the downstream signaling events. In addition, given that S904S does not lead to an amino acid substitution, the most plausible explanation for the previous results, would be the complete linkage with G691S. Nevertheless, it has been also proposed that S904S might influence RET expression by affecting RNA stability (19). Unfortunately, given that the clinical diagnosis for some members of the same family are conditioned on the time of genetic diagnosis of the corresponding proband, and that some members may have developed the disease immediately before the study was performed, the age at the onset of the disease is only truly known for the index cases. Because of the small sample size of our MEN 2A probands, the correlation between the age of onset and the polymorphisms/haplotypes could not be reliably performed. However, we could examine the effect of both variants in the appearance of PC/HPT, but no association was found, in agreement with previous reports (19).

In addition, we have analyzed S836S and the three intronic IVS1-1463T $\rightarrow$ C, IVS1-1370C $\rightarrow$ T and IVS1-126G $\rightarrow$ T, because of the association of such variants themselves or of specific haplotypes comprising them, to the sporadic forms of MTC or PTC $(28,33,34,40,41)$, which makes them good candidate variants to act as a modifier loci for MEN 2. Moreover, we have also selected two SNPs at -5 and -1 from the transcriptional start site $(-200 \mathrm{~A} \rightarrow \mathrm{G}$ and $-196 \mathrm{C} \rightarrow \mathrm{A})$, given that results derived from luciferase expression assays have shown differential activities for their different combinations (AC, GC and GA), suggesting a role of those variants in the transcriptional regulation of $R E T(29,42,43)$. Thus, we postulated that the two polymorphisms may also exert a modifier effect on MEN 2 phenotypes. Nevertheless, none of the RET variants here tested, nor any specific combination of them were found to be associated with the presence of PC and/or HPT. Our results, however do not exclude the possible role of other RET variants in the final presentation of the disease. Further studies are warranted in order to elucidate if other polymorphic variants located both in RET and other candidate genes may act as modifier elements of the disease.

\section{Acknowledgements}

We would like to thank the families who participated in the study. This study was funded by Fondo de Investigación Sanitaria, Spain (PI040266), Instituto de Salud Carlos III, Spain (C03/05), and Consejeria de Salud of Comunidad Autonoma de Andalucia (CAA 21/04).

\section{References}

1. Schimke RN: Genetic aspects of multiple endocrine neoplasia. Annu Rev Med 35: 25-31, 1984.

2. Eng C: RET proto-oncogene in the development of human cancer. J Clin Oncol 17: 380-393, 1999.

3. Gorlin RJ, Sedano HO, Vickers RA and Cervenka J: Multiple mucosal neuromas, phaeochromocytoma and medullary thyroid carcinoma of the thyroid: a syndrome. Cancer 22: 293-299, 1968.

4. Farndon JR, Leight GS, Dilley WG, Baylin SB, Smallridge RC, Harrison TS and Wells SA Jr: Familial medullary thyroid carcinoma without associated endocrinopathies: a distinct clinical entity. Br J Surg 73: 278-281, 1986.

5. Eng C, Clayton D, Schuffenecker I, et al: The relationship between specific RET proto-oncogene mutations and disease phenotype in multiple endocrine neoplasia type 2. International RET mutation consortium analysis. JAMA 276: 1575-1579, 1996.

6. Hansford JR and Mulligan LM: Multiple endocrine neoplasia type 2 and RET: from neoplasia to neurogenesis. J Med Genet 37: 817-827, 2000.

7. Asai N, Iwashita T, Matsuyama M and Takahashi M: Mechanism of activation of the ret proto-oncogene by multiple endocrine neoplasia 2A mutations. Mol Cell Biol 15: 1613-1619, 1995.

8. Borrello MG, Smith DP, Pasini B, et al: RET activation by germline MEN2A and MEN2B mutations. Oncogene 11: 2419-2427, 1995.

9. Santoro M, Carlomagno F, Romano A, et al: Activation of RET as a dominant transforming gene by germline mutations of MEN2A and MEN2B. Science 267: 381-383, 1995. 
10. Sanchez B, Robledo M, Biarnes J, et al: High prevalence of the C634Y mutation in the RET proto-oncogene in MEN 2A families in Spain. J Med Genet 36: 68-70, 1999.

11. Dang GT, Cote GJ, Schultz PN, Khorana S, Decker RA and Gagel RF: A codon 891 exon 15 RET proto-oncogene mutation in familial medullary thyroid carcinoma: a detection strategy. Mol Cell Probes 13: 77-79, 1999.

12. Iwashita T, Kato M, Murakami H, et al: Biological and biochemical properties of Ret with kinase domain mutations identified in multiple endocrine neoplasia type $2 \mathrm{~B}$ and familial medullary thyroid carcinoma. Oncogene 18: 3919-3922, 1999.

13. Pasini A, Geneste O, Legrand P, et al: Oncogenic activation by two distinct FMTC mutations affecting the tyrosine kinase domain. Oncogene 15: 393-402, 1997.

14. Kitamura Y, Scavarda N, Wells SA Jr, Jackson CE and Goodfellow PJ: Two maternally derived missense mutations in the tyrosine kinase domain of the RET proto-oncogene in a patient with de novo MEN2B. Hum Mol Genet 5: 1987-1988, 1995.

15. Smith DP, Houghton $C$ and Ponder BA: Germline mutation of RET codon 883 in two cases of de novo MEN 2B. Oncogene 15: 1213-1217, 1997.

16. Takahashi M: The GDNF/RET signaling pathway and human diseases. Cytokine Growth Factor Rev 12: 361-373, 2001.

17. Ponder BA: The phenotypes associated with ret mutations in the multiple endocrine neoplasia type 2 syndrome. Cancer Res 59: 1736-1741, 1999

18. Gil L, Azanedo M, Pollan M, et al: Genetic analysis of RET, GFR alpha 1 and GDNF genes in Spanish families with multiple endocrine neoplasia type 2A. Int J Cancer 99: 299-304, 2002.

19. Robledo M, Gil L, Pollan M, et al: Polymorphisms G691S/ S904S of RET as genetic modifiers of MEN 2A. Cancer Res 63: 1814-1817, 2003

20. Patocs A, Valkusz Z, Igaz P, Balogh K, Toth M, Varga I and Racz K: Segregation of the V804L mutation and S836S polymorphism of exon 14 of the RET gene in an extended kindred with familial medullary thyroid cancer. Clin Genet 63: 219-223, 2003.

21. Magalhaes PK, De Castro M, Elias LL, Soares EG and Maciel LM: Polymorphisms in the RET proto-oncogene and the phenotypic presentation of familial medullary thyroid carcinoma. Thyroid 14: 848-852, 2004.

22. Vanhorne JB, Andrew SD, Harrison KJ, et al: A model for GFR alpha 4 function and a potential modifying role in multiple endocrine neoplasia 2. Oncogene 24: 1091-1097, 2005.

23. Borrego S, Eng C, Sanchez B, Saez ME, Navarro E and Antiñolo G: Molecular analysis of the ret and GDNF genes in a family with multiple endocrine neoplasia type $2 \mathrm{~A}$ and Hirschsprung disease. J Clin Endocrinol Metab 83: 3361-3364, 1998.

24. Fernandez RM, Antiñolo G, Eng C and Borrego S: The RET C620S mutation causes multiple endocrine neoplasia type $2 \mathrm{~A}$ (MEN2A) but not Hirschsprung disease (HSCR) in a family cosegregating both phenotypes. Hum Mutat 22: 412-415, 2003.

25. Inabnet WB, Caragliano P and Pertsemlidis D: Pheochromocytoma: inherited associations, bilaterality, and cortex presentation Surgery 128: 1007-1011, 2000.

26. Dracapoli NH, Haines JL and Korf BR (eds): Current Protocols in Human Genetics. John Wiley \& Sons, New York, 1994.

27. Brandi ML, Gagel RF, Angeli A, et al: Guidelines for diagnosis and therapy of MEN type 1 and type 2. J Clin Endocrinol Metab 86: 5658-5671,2001

28. Borrego S, Wright FA, Fernandez RM, et al: A founding locus within the RET proto-oncogene may account for a large proportion of apparently sporadic Hirschsprung disease and a subset of cases of sporadic medullary thyroid carcinoma. Am J Hum Genet 72: 88-100, 2003.
29. Fernandez RM, Boru G, Peciña A, et al: Ancestral RET haplotype associated with Hirschsprung disease shows linkage disequilibrium breakpoint at -1249. J Med Genet 42: 322-327, 2005 .

30. Ceccherini I, Hofstra RM, Luo Y, et al: DNA polymorphisms and conditions for SSCP analysis of the 20 exons of the ret proto-oncogene. Oncogene 9: 3025-3029, 1994.

31. Borrego S, Saez ME, Ruiz A, Gimm O, Lopez-Alonso M, Antiñolo $G$ and Eng C: Specific polymorphisms in the RET proto-oncogene are over-represented in patients with Hirschsprung disease and may represent loci modifying phenotypic expression. J Med Genet 36: 771-774, 1999.

32. Borrego S, Ruiz A, Saez ME, et al: RET genotypes comprising specific haplotypes of polymorphic variants predispose to isolated Hirschsprung disease. J Med Genet 37: 572-578, 2000.

33. Ruiz A, Antiñolo G, Fernandez RM, Eng C, Marcos I and Borrego S: Germline sequence variant S836S in the RET protooncogene is associated with low level predisposition to sporadic medullary thyroid carcinoma in the Spanish population. Clin Endocrinol (Oxf) 55: 399-402, 2001.

34. Fernandez RM, Robledo M, Antiñolo G, Pecina A, RuizLlorente S, Eng C and Borrego S: The RET IVS1-126G $\rightarrow$ T variant is strongly associated with the development of sporadic medullary thyroid cancer. Thyroid 14: 329-331, 2004.

35. Saez ME, Ruiz A, Cebrian A, Morales F, Robledo M, Antiñolo G and Borrego S: A new germline mutation, R600Q, within the coding region of the RET proto-oncogene: a rare polymorphism or a MEN 2 causing mutation. Hum Mutat 15: 122, 2000.

36. Antiñolo G, Marcos I, Fernandez RM, Romero M and Borrego S: A novel germline point mutation, c. $2304 \mathrm{G} \rightarrow \mathrm{T}$, in codon 768 of the RET proto-oncogene in a patient with medullary thyroid carcinoma. Am J Med Genet 110: 85-87, 2002.

37. Pigny P, Bauters C, Wemeau JL, et al: A novel 9-base pair duplication in RET exon 8 in familial medullary thyroid carcinoma. J Clin Endocrinol Metab 84: 1700-1704, 1999.

38. Da Silva AM, Maciel RM, Da Silva MR, Toledo SR, De Carvalho MB and Cerutti JM: A novel germ-line point mutation in RET exon 8 [Gly(533)Cys] in a large kindred with familial medullary thyroid carcinoma. J Clin Endocrinol Metab 88: 5348-5343, 2003.

39. Gardner E, Mulligan LM, Eng C, Healey CS, Kwok JBJ and Ponder MA: Haplotype analysis of MEN 2 mutations. Hum Mol Genet 3: 1771-1774, 1994.

40. Gimm O, Neuberg DS, Marsh DJ, et al: Over-representation of a germline RET sequence variant in patients with sporadic medullary thyroid carcinoma and somatic RET codon 918 mutation. Oncogene 18: 1369-1373, 1999.

41. McWhinney SR, Boru G, Binkley PK, Peczkowska M, Januszewicz AA, Neumann HP and Eng C: Intronic single nucleotide polymorphisms in the RET proto-oncogene are associated with a subset of apparently sporadic pheochromocytoma and may modulate age of onset. J Clin Endocrinol Metab 88: 4911-4916, 2003

42. Fitze G, Appelt H, Konig IR, et al: Functional haplotypes of the RET proto-oncogene promoter are associated with Hirschsprung disease (HSCR). Hum Mol Genet 12: 3207-3214, 2003.

43. Burzynski GM, Nolte IM, Osinga J, et al: Localizing a putative mutation as the major contributor to the development of sporadic Hirschsprung disease to the RET genomic sequence between the promoter region and exon 2. Eur J Hum Genet 12: 604-612, 2004. 